\title{
IDENTIFICATION OF GeV ELECTRONS VIA PARTICLE MULTIPLICITY IN A SILICON DETECTOR: MEASUREMENT AND EGS SIMULATION
}

\author{
E. HEIJNE and P. JARRON \\ CERN, Geneva, Switzerland
}

\section{T. JENKINS and W.R. NELSON}

SLAC, Stanford, California, U.S.A.

\author{
H. ING \\ AECL, Chalk River, Ontario, Canada
}

Received 7 July 1982

The measurement of particle multiplicity in a silicon detector can be used to discriminate electrons in a flux of high energy particles. Monte Carlo simulations are compared with measurements at 4 and $15 \mathrm{GeV} / c$, for electrons and pions. With a $7 \mathrm{~mm}$ lead converter $80 \%$ of the electrons produce a signal higher than two minimum ionizing particles in a $63 \mathrm{~mm}^{2}$ silicon detector.

\section{Introduction}

When a flux of high energy particles is to be measured inside an absorber, the measurement can be complicated by the presence of secondary electrons. These may constitute a large fraction of the total number of counted particles when the incident particles have energies above $100 \mathrm{GeV}$. Especially in the case of muons, range and profile measurements in a shielding are subject to large errors if the electron contribution cannot be precisely determined. It is therefore of interest to develop a small counter which can measure a high flux of particles and can distinguish electrons from muons, pions, etc. To be useful for profile measurement in the high intensity beams which are now in operation, the counter should be much smaller than $1 \mathrm{~cm}^{2}$. Such a detector has been constructed in the form of a telescopic arrangement of silicon detectors and a lead converter of a few mm thickness.

A Monte Carlo simulation was performed using the electromagnetic cascade program EGS [1] for different geometries, and some of these geometries were used at SLAC in a test beam of 4 and $15 \mathrm{GeV} / c$ electrons and pions.

\section{Design of the detector telescope}

The telescope consists of 2 small silicon surface barrier diode detectors ${ }^{*}$ of $25 \mathrm{~mm}^{2}$ and $63 \mathrm{~mm}^{2}$, which are 385 and $486 \mu \mathrm{m}$ thick, respectively. Each of them is mounted directly on the preamplifier printed circuit board, as shown in figs. 1 and 2 . Concentric pieces of oxidized aluminium hold the detectors in place and provide space for a lead plug, which can be up to $7 \mathrm{~mm}$ thick. The overall thickness of the box is $21 \mathrm{~mm}$.

The purpose of this arrangement is to measure the flux with the smaller upstream diode and identify particles as electrons or muons (pions) using the coincident signal in the downstream detector. With the sketched geometry an acceptance angle of $40^{\circ}$ is obtained, which is much more than the test beam divergence.

\section{Electronics and experimental set-up}

The voltage sensitive preamplier has already been described in some detail [2]. It is designed for 50-100 $\mathrm{MHz}$ operation, so that it matches the charge collection time in a $300 \mu \mathrm{m}$ thick silicon detector. It was followed by a differentiating shaping amplifier of $3 \mathrm{~dB}$, which delivered a signal of $\sim 40 \mathrm{~ns}$ duration. Further amplification was done by standard NIM linear amplifiers. The system was calibrated and routinely monitored for stability using ${ }^{57} \mathrm{Co}$ and ${ }^{133} \mathrm{Ba}$ sources, and with a signal

\footnotetext{
* The detectors were purchased from Philips N.V., Eindhoven, The Netherlands
} 


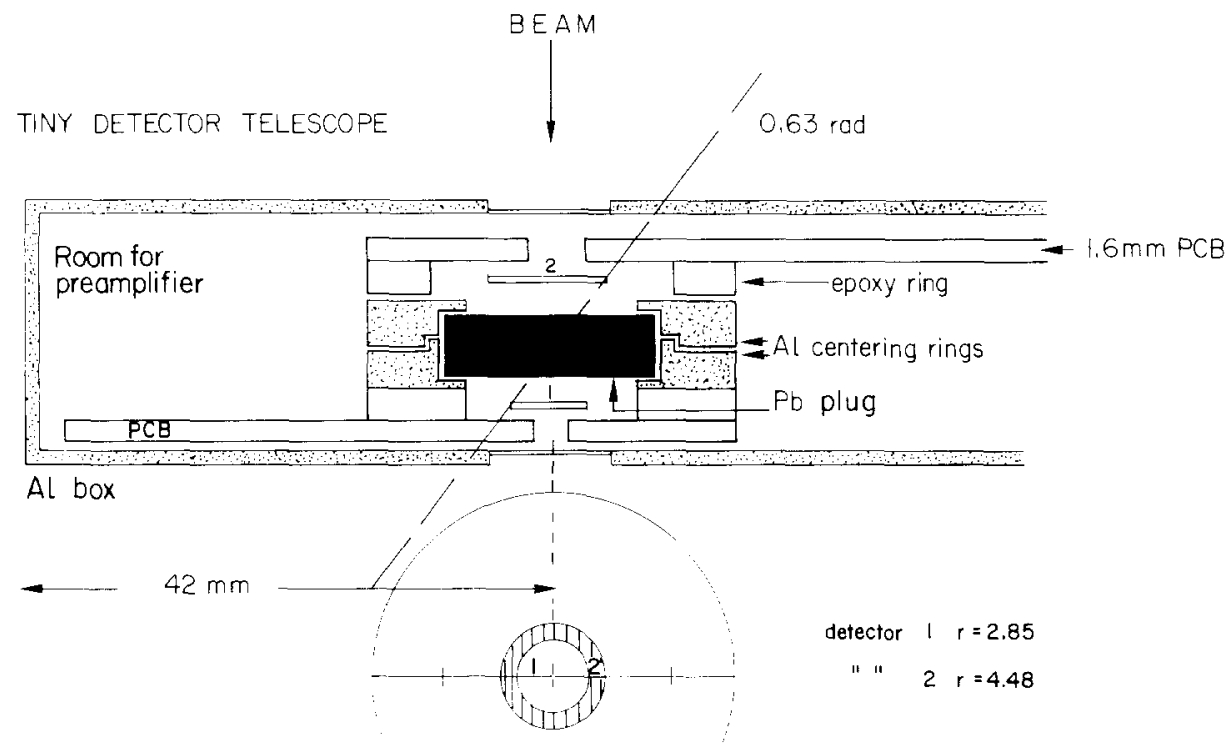

Fig. 1. Diagram of the detector box, showing the close mounting of all components. The printed circuit board (PCB) carries both the preamplifier and the detector.

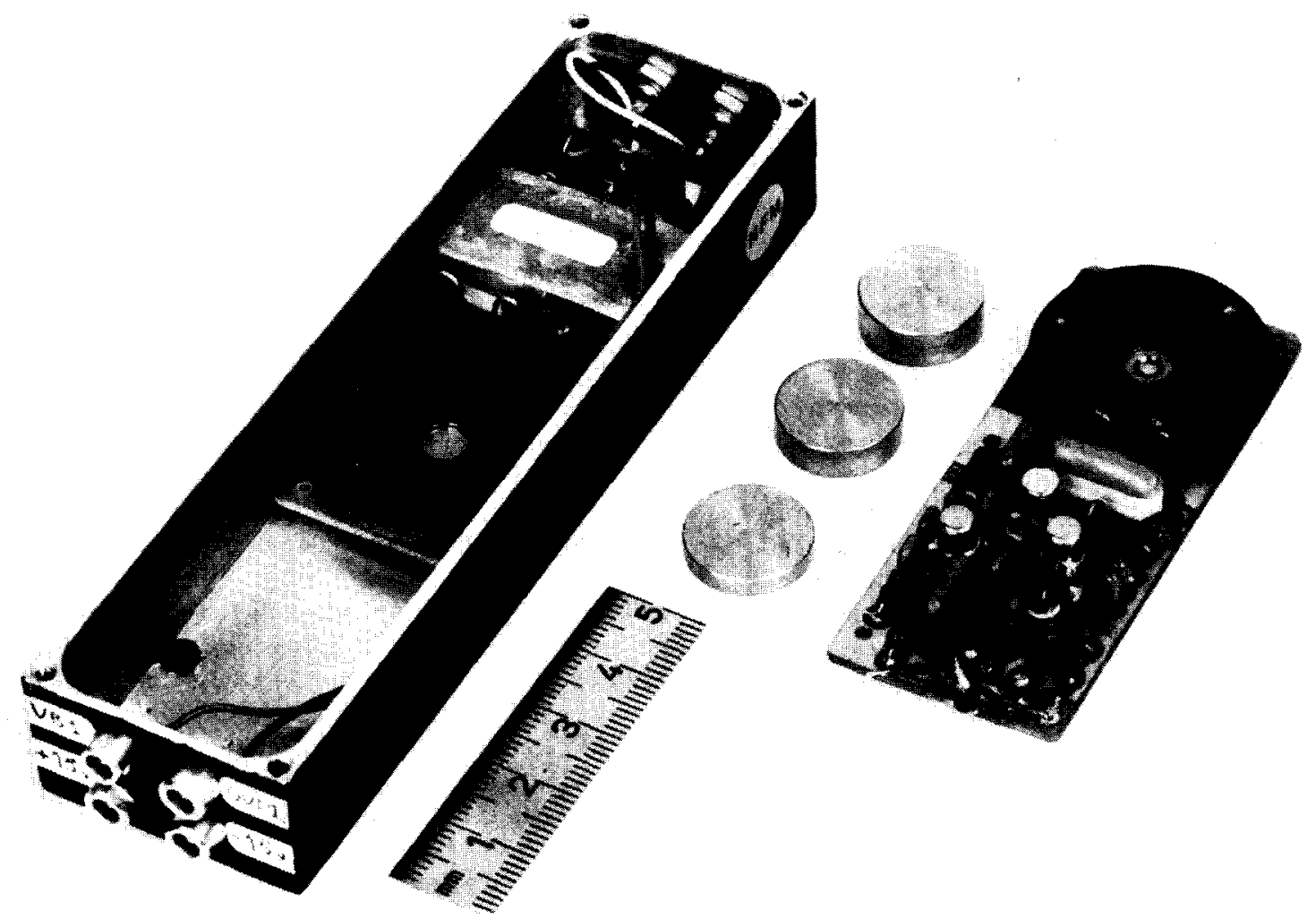

Fig. 2. Picture of the box containing the analyzing detector, the lead converters of 3,5 and $7 \mathrm{~mm}$ thickness and the upstream silicon detector, which has in this case a $1 \mathrm{~mm}^{2}$ area only. The rings keep the detectors precisely centered and attach them to the preamplifier circuit board. 


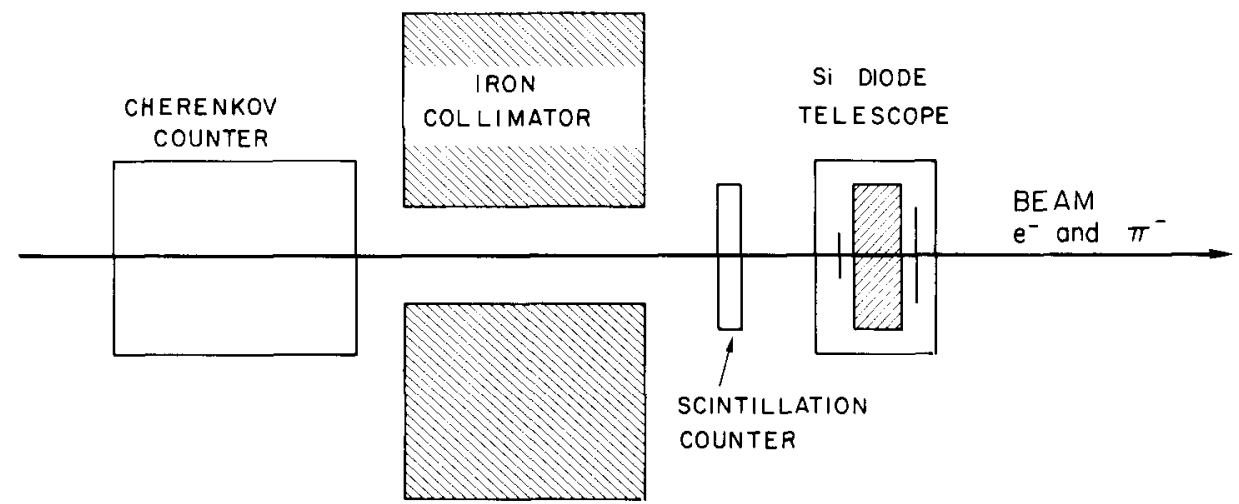

Fig. 3. Experimental set-up in Beam Line 6 at SLAC. The drawing is not to scale.

pulse generator. The pulse height was analyzed with a multichannel analyzer (Le Croy QVT 3001), which was gated by a logic signal that was a coincidence between the detector signal, a beam scintillator signal and a threshold Cherenkov counter (AND or NOT) signal. The experimental set-up in Beam Line 6 at SLAC is shown in fig. 3. Data were taken with secondary beam momenta of 4 and $15 \mathrm{GeV} / c(\Delta p / p=1.5 \% \mathrm{fwhm})$ produced by $20.5 \mathrm{GeV}$ primary electrons with a pulse width of $1.5 \mu \mathrm{s}$. The secondary beam dimensions after passing through a $91.5 \mathrm{~cm}$ long iron collimator with a $1 \mathrm{~cm}$ diameter aperture were $1 \mathrm{~cm}$ (uniform) horizontally and $5 \mathrm{~mm}$ (fwhm) vertically. The intensity was adjusted so that on the average there was less than one-third particle per beam pulse at a rate that varied between 10 and 70 pps. Beam Line 6 is a mixture of electrons and pions with a $\mathrm{K} / \pi$ ratio less than $10^{-2}$.

The $\mathrm{e} / \pi$ ratio has the capability of varying between 0.01 and 10 depending on the choice of momentum, target, and filter combination. In all cases the Cherenkov counter was tuned to identify electrons. The electron runs were done with an $\mathrm{e} / \pi \approx 0.5$ and by using the AND signal from the Cherenkov counter. The pion runs used the NOT signal and an $\mathrm{e} / \pi \approx 0.1$.

\section{EGS simulation}

Using the EGS code system [1] a simulation of the electron transmission through several thicknesses of lead was made, and the number of charged particles reaching the $63 \mathrm{~mm}^{2}$ detector through the lead plug was determined. This number is shown in fig. 4 as a function of the lead thickness for several incident electron energies. The geometry for this simulation is sketched in the insert of fig. 4. For $100 \mathrm{MeV}$ incident electrons, the probability of an electron reaching the detector surface within its radius is somewhat below one, regardless of the absorber thickness. For a detector with an infinitely large radius the probability is somewhat above one. Below $100 \mathrm{MeV}$ the probability drops rapidly. For example, the mean projected range for a $30 \mathrm{MeV}$ electron is $4.8 \mathrm{~mm}$ in lead [3], and multiple scattering at the end of the range diminishes the probability of giving a signal in the detector.

EGS simulations were done at 4 and $15 \mathrm{GeV}$. Charged particles reaching the detector surface within its radius were recorded on magnetic tape. The subsequent phase of the analysis consisted of approximating the energy deposition in the detector by sampling from a Landau distribution for each of the scoring particles. The mean energy loss was first determined from the detector thickness and the stopping power. Then the Landau distributed value was found with the routine DISLAN [4].

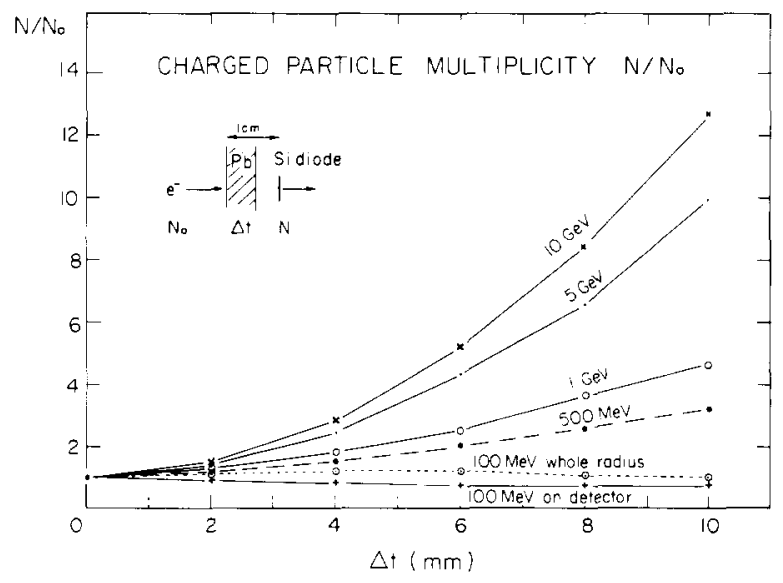

Fig. 4. Results of the EGS simulation of particle multiplicity $N$ in the detector for different energies of the incident electrons $N_{0}$ as a function of the lead thickness $\Delta t$. 


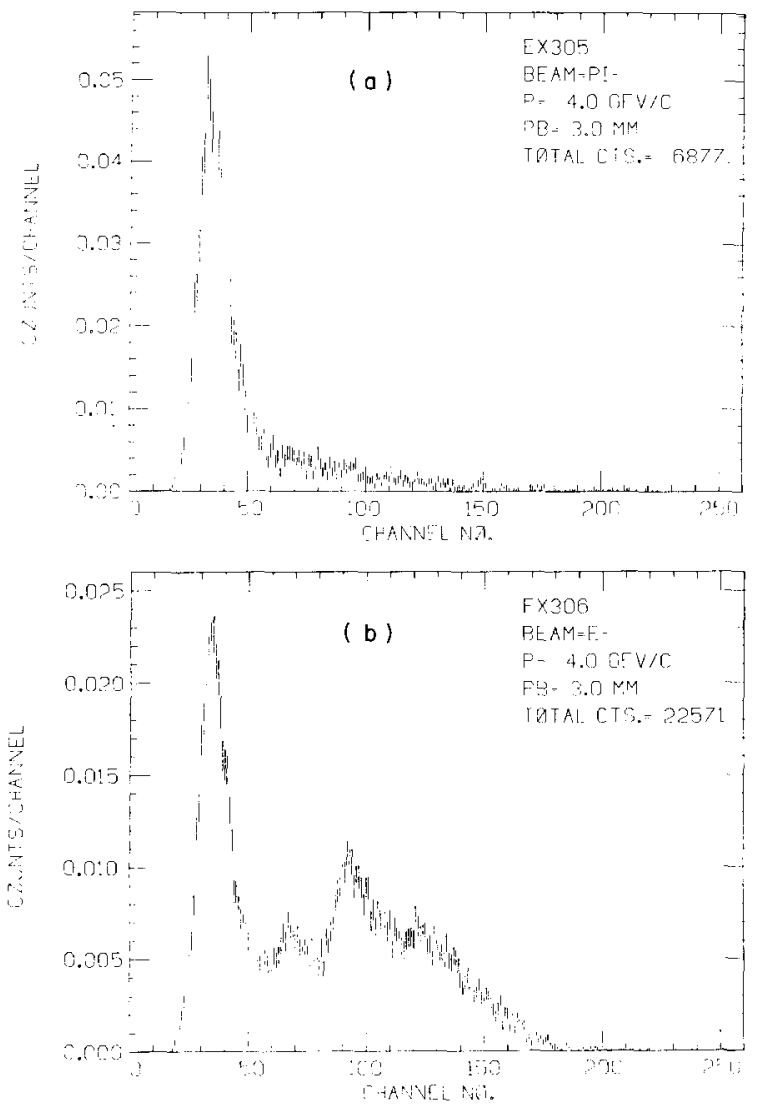

Fig. 5. Experimental spectra for $\pi^{-}$(run EX305) and $\mathrm{e}^{-}$(run EX306) for the same momentum $(4 \mathrm{GeV} / \mathrm{c})$ and the same converter thickness $(3.0 \mathrm{~mm})$.
Finally, the results were plotted as a pulse-height spectrum, comparable to the one produced by the multichannel analyzer.

\section{Results of the measurements}

In table 1 a summary is given of the energies and absorber thicknesses used in both the pion and electron runs. The pulse height spectra obtained for the pions do not show notable multiplicity (e.g., see fig. 5a), and the number of counts with a pulse height beyond twice the most probable value is less than $\sim 20 \%$ [table 1 , column (e)]. A slight increase of the tail, from $14 \%$ to $23 \%$, can be noted for the $4 \mathrm{GeV} \pi^{-}$, with increasing lead thickness. This can be attributed to pion interactions, and it should be less in the case of muons. The number of secondary electrons $>10 \mathrm{MeV}$ generated by a $15 \mathrm{GeV}$ pion or muon in $5 \mathrm{~mm}$ of lead is only $\sim 0.03$. The electron spectra taken without any lead in front of the analyzing detector are identical to the pion spectra (with or without lead).

In figs. $5 \mathrm{a}$ and $5 \mathrm{~b}$ the $\pi^{-}$spectrum for $4 \mathrm{GeV} / c$ and $3 \mathrm{~mm}$ lead is compared with the corresponding spectrum for $\mathrm{e}^{-}$. For incident electrons, $58 \%$ of the events produces signals in the second detector greater than twice the most probable value. For 5 and $7 \mathrm{~mm}$ of lead this proportion increases to $77 \%$ and $84 \%$, respectively.

A few more experimental spectra are shown in figs. $6 a, b, c$, together with the results of the EGS simulations in figs. $6 \mathrm{~d}, \mathrm{e}, \mathrm{f}$, which were made with approximately the same number of events and are represented on the same scale as the measured pulse height spectra. It is

Table 1

Summary of experimental runs.

\begin{tabular}{|c|c|c|c|c|c|}
\hline $\begin{array}{l}\text { (a) } \\
\text { Run }\end{array}$ & $\begin{array}{l}\text { (b) } \\
\text { Particle }\end{array}$ & $\begin{array}{l}\text { Momentum } \\
(\mathrm{GeV} / c)\end{array}$ & $\begin{array}{l}\text { (d) } \\
\text { Converter } \\
\text { thickness } \\
(\mathrm{mm})\end{array}$ & $\begin{array}{l}\text { (e) } \\
\text { Percentage of } \\
\text { events with signal } \\
>2 \text { m.p.v. }{ }^{\text {h) }}\end{array}$ & $\begin{array}{l}\text { (f) } \\
\text { Apparent average } \\
\text { multiplicity }\end{array}$ \\
\hline EX302 & $\pi^{-}$ & 4 & 0. & 14. & 1.3 \\
\hline EX305 a) & $\pi^{-}$ & 4 & 3. & 18. & 1.5 \\
\hline EX304 & $\pi^{-}$ & 4 & 7. & 23. & 1.7 \\
\hline EX309 & $\pi^{-}$ & 15 & 5. & 20. & 1.6 \\
\hline EX301 & $\mathrm{e}^{-}$ & 4 & 0. & 15. & 1.4 \\
\hline $\mathrm{EX} 306^{\mathrm{a})}$ & $\mathrm{e}^{-}$ & 4 & 3. & 58. & 2.5 \\
\hline $\mathrm{EX} 307^{\mathrm{a})}$ & $\mathrm{e}^{-}$ & 4 & 5. & 77. & 3.4 \\
\hline EX303 & $\mathrm{e}^{-}$ & 4 & 7. & 84. & 3.9 \\
\hline $\mathrm{EX} 310^{a)}$ & $\mathrm{e}^{-}$ & 15 & 5. & 88. & 4.3 \\
\hline
\end{tabular}

a) Also, see figs. 5 and/or 6 .

b) Most probable value of single multiplicity peak. 
obvious that the odd multiplicities $(1,3,5, \ldots)$ are more frequent than the even $(2,4, \ldots)$. This can be attributed to the fact that bremsstrahlung and pair production mechanisms dominate at these high energies. Beyond a multiplicity of 5 (4 in the case of $15 \mathrm{GeV} / c$ ) it is impossible to distinguish peaks any more in the experi-

\section{EXPERIMENT}
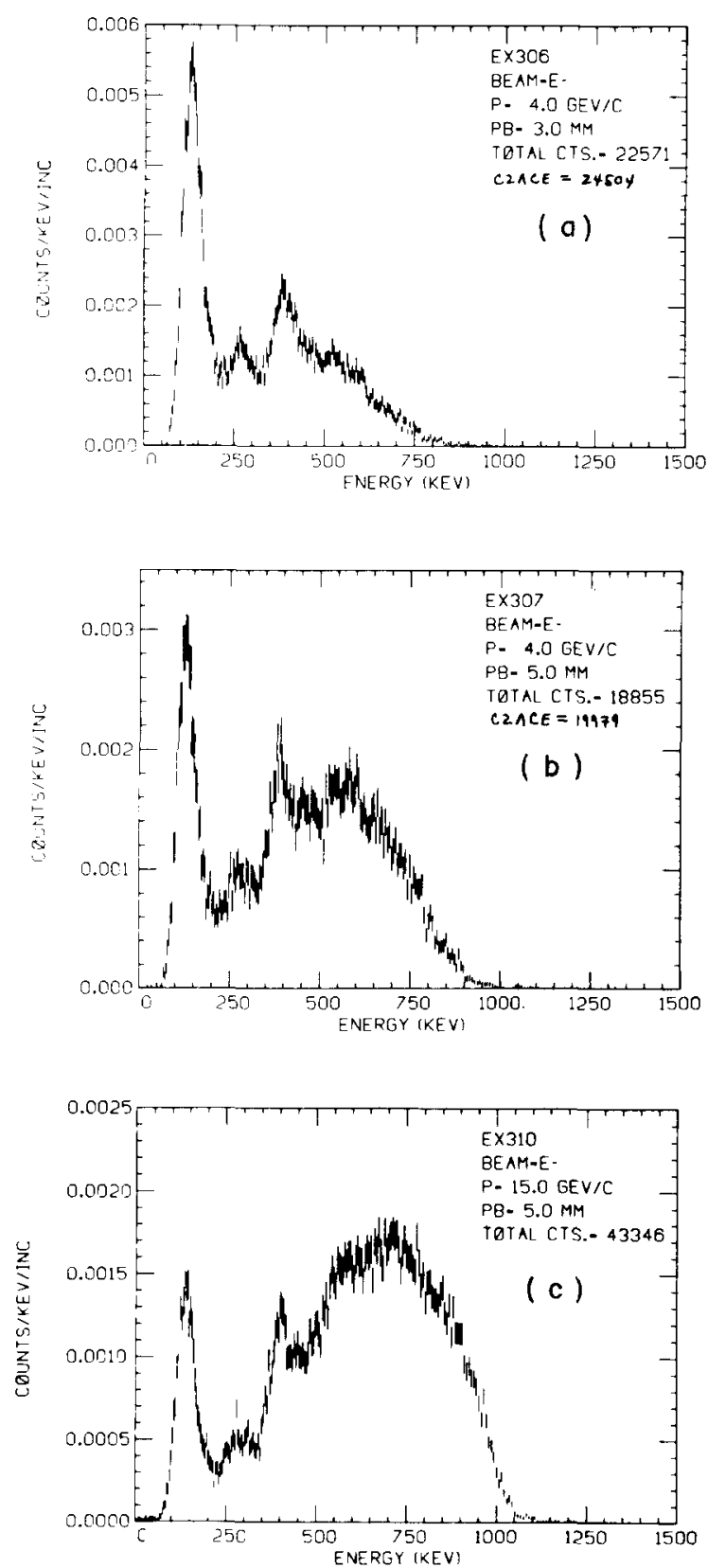

mental distributions and even in the simulated distributions they are hardly visible.

The pulse height spectrum beyond $\sim 1 \mathrm{MeV}$ drops rather abruptly on the side of high multiplicities. At first glance, this effect resembles electronic saturation. However, this is unlikely because pulses up to an equiv-
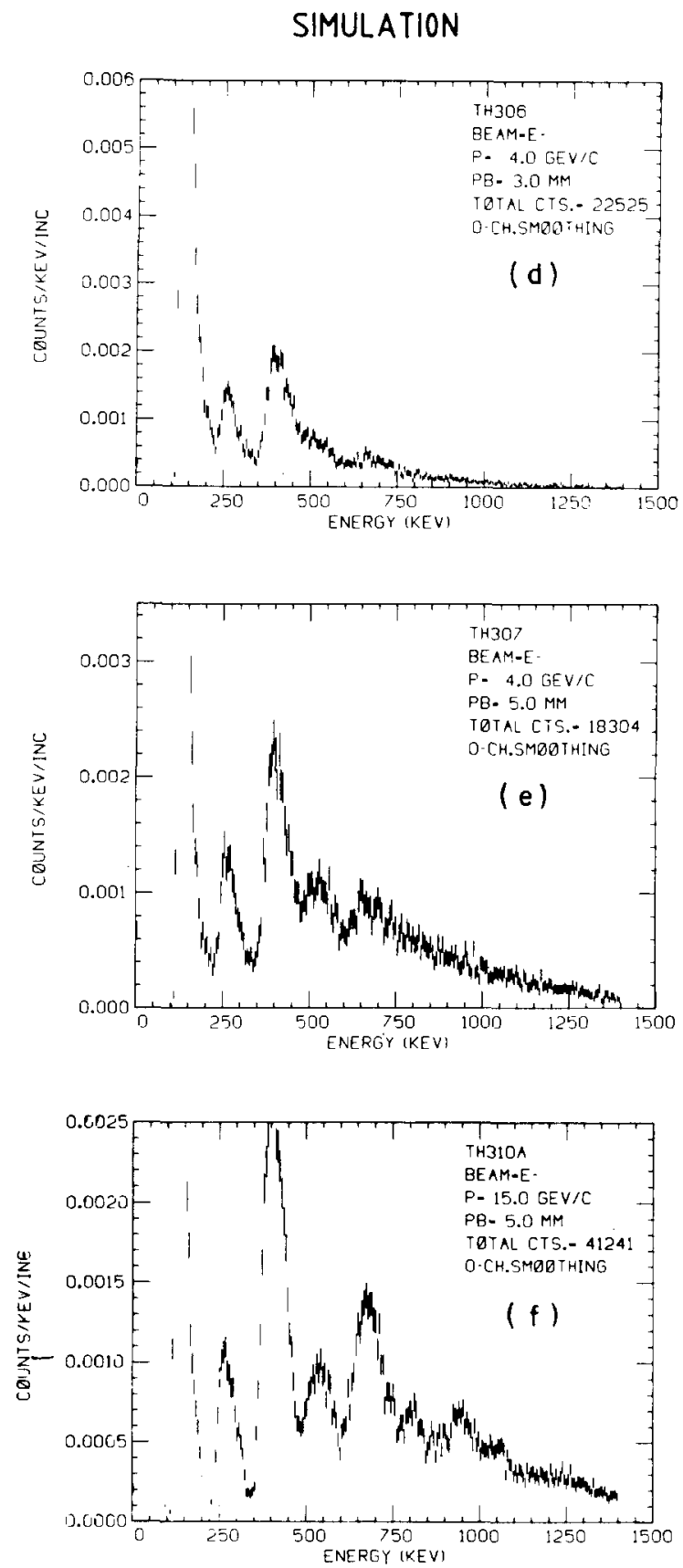

Fig. 6. Comparison of the experimental spectra EX306, EX307 and EX310 (left) with the corresponding EGS/Landau simulations (right). 
alent of $1.5 \mathrm{MeV}$, corresponding to the maximum range of the ADC, were normally processed without saturation. In fact, the high energy "edges" occur at different energies for the different runs shown in figs. $6 a, b, c$, and they are higher for the higher electron energy and for the thicker lead absorbers.

Using the absolute calibration with the radioactive sources, the most probable value (m.p.v.) of the energy deposition in the detector for a minimum ionizing particle (i.e., the single multiplicity peak) was found to be $137 \mathrm{keV}$, which agrees to $3 \%$ with the expected value of $29 \mathrm{keV}$ per $100 \mu \mathrm{m}$ of Si [5].

Dividing the energy scale in equal bins, centered around the positions of $1,2,3, \ldots$ times the "m.p.v." of the single multiplicity peak, an average apparent multiplicity can be calculated for each spectrum [table 1, column (f)].

\section{Discussion}

Although the qualitative agreement between the simulation and the measurement is quite satisfactory, there are two obvious discrepancies. The experimental spectra show fewer events of low multiplicity than predicted by the calculations. This may be explained by the fact that the simulation did not account for the detection of photons from the shower by the detector. For low energy photons (below $\sim 100 \mathrm{keV}$ ), the response to photons (in coincidence with the electrons of the shower) may not be negligible and these pulses would sum with electron signals throwing them into a region of higher apparent multiplicity.

The measured spectrum also drops off more abruptly than the calculated spectrum. The latter, obtained using the Landau distribution, actually represents the spectrum of energies lost by the electrons in traversing the detector. However, all these energies may not be collected completely in the detector because many of the secondary electrons, generated in the detector, escape before depositing all their energies. This effect becomes increasingly important as the multiplicity increases, causing the measured spectrum to skew towards lower energy. Detailed comparison of the measured and calculated spectra would require a better knowledge of the detector response function for both electrons and photons.

In spite of the details, it can be concluded that in $70-90 \%$ of the events, high energy electrons can be identified. A known fraction of $15-20 \%$ of the pions or muons give a signal above the 2 "m.p.v." threshold due to the Landau energy distribution. With less than 10 $\mathrm{mm}$ of lead converter, incident electrons with energy bectween $100 \mathrm{MeV}$ and $1 \mathrm{GeV}$ are difficult to identify with the present telescope, since quite frequently only one particle will reach the second (multiplicity) detector. A possible solution for this is to include a measurement of the multiple scattering, using a multi-element detector like the silicon microstrip detector [6]. A further advantage of such a detector is that its very small elements (e.g., $0.1 \mathrm{~mm}^{2}$ ) enable a sufficiently low count rate even in a high flux.

A possible application of this device would be in the muon flux measurement in the Tevatron neutrino beam line.

We wish to thank R. Gearhart for his help with Beam Line 6 and the SLAC accelerator operations personnel.

\section{References}

[1] R.L. Ford and W.R. Nelson, The EGS code system: SLAC report No. 210. Stanford (1978).

[2] E.H.M. Heijne et al., IEEE Trans. Nucl. Sci.NS-27 (1980) 272.

[3] T. Tabata, R. Ito, S. Okabe and Y. Fujita, J. Appl. Phys. 42 (1971) 3361.

[4] B. Schorr, CERN Computer Centre, Long write-up G110/G111, Geneva (1974).

[5] E. Heijne, P. Jarron and G. Viertel, to be published.

[6] E.H.M. Heijne, et al., Nucl. Instr. and Meth. 178 (1980) 331. 